\section{Allergologie für Anfänger}

D ie Autoren wollen mit dem hier vorgestellten Werk eine erste „Hilfestellung für Einsteiger" in Diagnostik und Therapie der Allergien bieten. Tatsächlich ist das Buch recht übersichtlich gehalten - allerdings sparsam illustriert - und kann besonders bei methodischen Fragen und im Kapitel „Laboruntersuchungen“ punkten.

Das Lehrbuch behandelt recht knapp die Grundlagen der Sensibilisierung und der allergischen Reaktionsweisen. Die wichtigsten allergologischen Krankheitsbilder werden zwar auch mit klinischem Bild und ihrer In-vivo-Diagnostik umrissen, mög- liche Differenzialdiagnosen und das breite Spektrum des therapeutischen Vorgehens kommen allerdings etwas kurz. Auch werden die Überschneidungen zwischen allergischen und nicht allergischen Unverträglichkeitsreaktionen kaum erwähnt. Die symptomatische medikamentöse und die kausale spezifische Immuntherapie sowie das Vorgehen bei allergologischen Notfällen sind für den Anfänger ausreichend erörtert, das Buch könnte aber für die weitere Vertiefung Literaturhinweise und Internet-Adressen bereithalten.

Insgesamt gesehen bietet das Buch für Einsteiger und vor allem auch für an labormethodischen Fragen Interessierte eine aktuelle Darstellung, es gibt aber die Breite der praktischen klinischen Allergologie nicht so umfassend wieder, wie man es sich gerade auch für den zweiten Blick eines Anfängers wünscht.

\section{Dr. Ulrich Mutschler, Hildesheim}

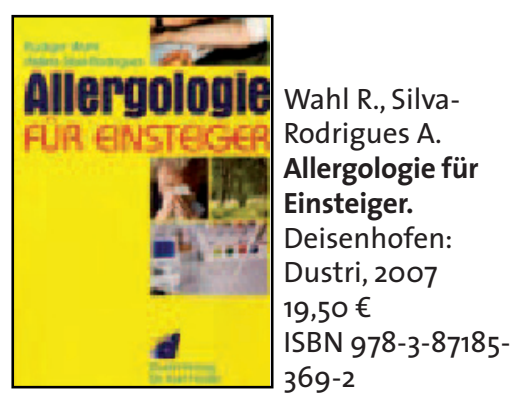

kann sicher sein, dass es das ganze Fachgebiet in seinem konservativen Bereich abdeckt.

Prof. Dr. Olaf Michel, Brüssel über die Glossodynie bis hin zur Lepra -, werden ebenso abgehandelt wie die lokale und sytemische antibiotische Therapie der entzündlichen HNO-Erkrankungen, einer Spezialität des Autors. Luckhaupt greift aber auch eine alte HNO-Tradition auf und bringt viele Rezepturen - die Apotheker werden sich freuen, sie bleiben in Übung.

Allein wegen der Aktualität ist dieses Buch zu empfehlen und man

\title{
Panacea der HNO-Heilkunde
}

$\mathrm{H}$ ans-Peter Zenner hat mit dieser zweiten Auflage des Standardwerks der Therapie von HNO-Erkrankungen die schon ausgezeichnete erste Auflage noch übertroffen. „Praktische Therapie von HNO-Krankheiten " ist Nachschlagewerk und systematisches Lehrbuch, praktische Arbeitsanweisung und Quelle für Geheimtipps. Selbst wenn auch dieses Buch das lang ersehnte "Panacea“ (Allheilmittel der Alchemie) nicht beschreibt, es enthält doch alle Möglichkeiten der modernen Be- handlung von Erkrankungen im HNOBereich.

Das Werk ist reich bebildert und ermöglicht jederzeit den Bezug zur täglichen HNO- und allgemeinärztlichen Praxis. Besonders positiv fallen die praktischen Arbeitsmaterialien auf, wie Rezepturen, Anleitungen für die Therapie und Therapieschemata, Aufklärungsbögen, Übungsschemata für Patienten und vieles mehr.

Somit ist "Praktische Therapie von HNO-Krankheiten“ ein Lehrbuch der Therapie für HNO-Ärzte, HNOWeiterbildungsassistenten, für Allgemeinmediziner, Internisten und Chirurgen.

Prof. Dr. Ludger Klimek, Wiesbaden

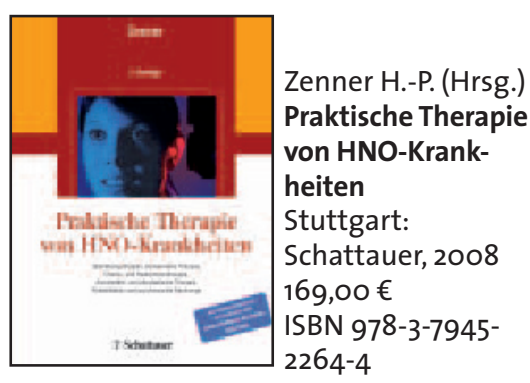

